Revue Française de Civilisation Britannique

\title{
La création d'emplois en Grande-Bretagne dans les années 1990
}

Job Creation in Britain in the 1990 s

Martine Azuelos

\section{CpenEdition}

Journals

Édition électronique

URL : http://journals.openedition.org/rfcb/3117

DOI : $10.4000 /$ rfcb. 3117

ISSN : 2429-4373

Éditeur

CRECIB - Centre de recherche et d'études en civilisation britannique

Édition imprimée

Date de publication : 1 avril 2003

ISBN : 2-911580-16-8

ISSN : 0248-9015

Ce document a été généré automatiquement le 31 mai 2019.

\section{(c)}

Revue française de civilisation britannique est mis à disposition selon les termes de la licence Creative Commons Attribution - Pas d'Utilisation Commerciale - Pas de Modification 4.0 International. 


\section{La création d'emplois en Grande- Bretagne dans les années 1990}

Job Creation in Britain in the 1990s

Martine Azuelos

1 Alors que les médias français opposaient volontiers, dans les années 1990, la facilité avec laquelle on pouvait trouver du travail en Grande-Bretagne aux dures réalités de l'insertion professionnelle dans notre pays, la plupart des travaux universitaires consacrés à l'évolution du marché du travail britannique pendant la même période ont quant à eux mis l'accent, tantôt sur la progression du chômage, tantôt sur le développement de formes d'emplois atypiques allant de pair avec des situations de précarité. Peu de travaux se sont attachés à étudier la création d'emplois, et quand celleci a été évoquée, on a souvent insisté sur le fait que ce phénomène, ainsi que la résorption du chômage observée entre la fin de la récession du début des années 1990 et la fin de la décennie, résultaient principalement du développement de l'emploi précaire, à temps partiel et mal rémunéré. Ces évolutions ont par ailleurs été mises en rapport avec la disparition massive d'emplois industriel - emplois à temps plein, stables et relativement bien rémunérés du fait de la forte syndicalisation historique de ce secteur - et le développement d'une flexibilité largement imputable aux réformes introduites par les conservateurs pendant les années 1980 .

2 Les gouvernements conservateurs en place de 1979 à 1997 ont pour leur part insisté sur le fait que, grâce aux réformes mises en œuvre depuis l'arrivée au pouvoir de Margaret Thatcher, la Grande-Bretagne était devenue plus fortement créatrice d'emplois et surpassait, dans ce domaine, bon nombre de pays de l'Union européenne. Ce constat rejoignait celui que dressait l'OCDE en 2000 lorsque l'organisation soulignait, dans son étude économique sur le Royaume-Uni ${ }^{1}$, que ce pays avait « été (...) l'un des pays les plus actifs dans la mise en œuvre de la stratégie pour l'emploi », ajoutant que cette politique semblait « porter ses fruits $»^{2}$ Ce faisant, le marché du travail britannique se serait de plus en plus rapproché, dans ses modes de fonctionnement, du marché du travail américain, et éloigné des marchés du travail européens, et en particulier du modèle français, traditionnellement soucieux de la protection sociale des salariés et fortement réglementé 
par les pouvoirs publics. Il est d'ailleurs intéressant de relever que les jugements pessimistes souvent portés, outre-Manche, sur les évolutions récentes du marché du travail britannique rejoignent ceux que l'on entendait fréquemment, outre-Atlantique, dans les années 1970 et 1980, à un moment où les États-Unis, pourtant entrés dans une phase de ralentissement économique, créaient massivement des emplois. Ces emplois, disait-on alors, étaient le plus souvent des 'Mac Jobs' mal payés, précaires, à temps partiel et n'offrant aucune perspective d'avenir ('dead-end jobs'). Or les études récentes menées sur les créations d'emplois aux États-Unis montrent que si le développement de certaines formes de précarisation est indéniable et si le salaire horaire moyen a baissé de façon continue du début des années 1970 à $1996^{3}$, l'ensemble de la période a été caractérisé par une progression sensible de l'emploi qualifié ${ }^{4}$.

La diversité des jugements portés sur la création d'emplois ne peut donc manquer d'inciter l'observateur français à tenter de mieux tenter de cerner un phénomène dont il perçoit, d'emblée, la complexité. On commencera ici par dresser un état des lieux en mesurant l'ampleur et la nature de la création d'emplois en Grande-Bretagne dans les années 1990, en replaçant cette décennie dans le contexte des évolutions à plus long terme qui sont observables depuis la fin des années 1960 et en tentant de dégager la spécificité des évolutions britanniques par rapport à celles qui ont pu être constatées en France et aux États-Unis. Ce faisant, on mettra en évidence à la fois le volume de la création d'emplois et son rythme de progression (première partie), ainsi que les secteurs et les types d'emplois qui en ont le plus largement bénéficié (deuxième partie). Enfin, dans une troisième partie, on s'attachera à identifier les principales forces qui peuvent expliquer les phénomènes observés.

\section{La mesure du phénomène : les grandes tendances}

L'un des avantages d'une étude sur la création d'emplois, par rapport à une étude sur le chômage, c'est qu'elle pose moins de problèmes statistiques: on sait que les modes de comptabilisation du chômage en Grande-Bretagne ont souvent changé depuis les année 1980, ce qui est une source de difficultés majeures quand on veut établir des séries statistiques pour étudier l'évolution du phénomène dans le temps ${ }^{5}$. Par ailleurs la définition de la situation de "chômeur » est elle-même de plus en plus problématique à une époque où les frontières traditionnellement étanches entre inactivité et activité tendent à s'estomper. En matière de création d'emplois, les choses sont un peu plus simples, même si l'observation des chiffres bruts doit être nuancée par la prise en compte de la typologie de l'emploi créé.

Comme le montrent les tableaux 1, 2 et 3, la création d'emplois, faible dans les années 1960, négative dans les années 1970, a repris fortement dans les années 1980 et s'est poursuivie, quoiqu'à un rythme moitié moins soutenu, dans les années $1990^{7}$. 
Tableau 1. Population active occupée au Royaume-Uni, 1961-2000 (en milliers)

\begin{tabular}{|l|l|l|l|l|}
\hline 1961 & 1971 & 1981 & 1991 & $2000^{*}$ \\
\hline 25132 & 25213 & 25140 & 27118 & 28118 \\
\hline
\end{tabular}

* chiffres enregistrés au printemps

Source: Duncan MC GREGOR, « Jobs in the Public and Private Sectors », Economic Trends, N 559, juin 2000, p. 34; Labour Market Trends, janvier 2001.

Tableau 2. Croissance de l'emploi au Royaume-Uni, 1961-2000

\begin{tabular}{|l|l|l|l|l|l|l|l|}
\hline & $1961-1971$ & $\begin{array}{l}1971- \\
1981\end{array}$ & $\begin{array}{l}1981- \\
1991\end{array}$ & $\begin{array}{l}1991- \\
2000\end{array}$ & $\begin{array}{l}1961- \\
1981\end{array}$ & $\begin{array}{l}1981- \\
2000\end{array}$ & $\begin{array}{l}1961- \\
2000\end{array}$ \\
\hline En milliers & 81 & -73 & 1978 & 1000 & 8 & 2978 & 3668 \\
\hline En \% & 0,3 & $-0,3$ & 7,9 & 3,7 & 0,03 & 11,8 & 15,0 \\
\hline En \% annuel moyen & 0,03 & $-0,03$ & 0,8 & 0,4 & 0,001 & 0,6 & 0,5 \\
\hline
\end{tabular}

Source : calculs de l'auteur d'après les données du tableau 1.

6 Le travail d'harmonisation réalisé par les statisticiens de l'OCDE dans le cadre de l'Étude sur l'emploi publiée par cet organisme en 1994 permet de comparer les évolutions enregistrées au Royaume-Uni avec celles qui furent enregistrées en France et aux ÉtatsUnis de 1979 à 1990. Il n'existe malheureusement pas, à ce jour, d'étude semblable pour la période 1991-2000. Pour la période antérieure, toutefois, on relève que la croissance de l'emploi au Royaume-Uni a été beaucoup moins forte qu'aux États-Unis, et beaucoup plus forte qu'en France. Les données de la colonne concernant la croissance de l'emploi par habitant sont à cet égard particulièrement intéressantes, puisqu'elles révèlent une croissance négative en France et une progression six fois plus faible au Royaume-Uni qu'aux États-Unis.

Tableau 3. Croissance de l'emploi au Royaume-Uni, aux États-Unis et en France, 1979-1990 (en \% annuel moyen)

\begin{tabular}{|l|l|l|}
\hline Pays & Croissance de l'emploi & $\begin{array}{l}\text { Croissance de l'emploi } \\
\text { par habitant }\end{array}$ \\
\hline Royaume-Uni & 0,5 & 0,1 \\
\hline États-Unis & 1,6 & 0,6 \\
\hline France & 0,2 & $-0,6$ \\
\hline
\end{tabular}

Source: OCDE, Étude sur l'emploi, 1994, vol. 1, p. 3-4. 
7 Bien entendu, il s'agit ici de créations nettes (intégrant tout à la fois les créations et les suppressions d'emplois), qui cachent ce que les économistes appellent parfois de fortes "turbulences", induites par la recomposition du paysage économique et social. Cette recomposition résulte elle-même de facteurs tels que la création et la disparition d'entreprises, ou des embauches et des licenciements pratiqués par les employeurs en fonction de la conjoncture, ou encore du caractère plus ou moins porteur de leur activité. Une étude publiée par le Centre for Economic Performance de la London School of Economics pour les années 1980 permet de bien mesurer l'ampleur de ce phénomène sur cette période ${ }^{8}$. En l'absence d'une étude du même type portant sur les années 1990, nous pouvons faire l'hypothèse que celui-ci a perduré. L'étude de l'OCDE citée précédemment nous permet en outre de comparer l'évolution de la répartition de l'emploi dans les principaux secteurs d'activité dans les trois pays (voir tableau 4).

8 On relève des évolutions comparables à certains égards : dans les trois pays, l'agriculture et l'industrie manufacturière ont supprimé des emplois et l'essentiel de la création d'emplois s'est produite dans les services. Notons toutefois que le déclin de l'emploi industriel a été beaucoup plus marqué au Royaume-Uni que dans les deux autres pays. Qui plus est, à l'intérieur du secteur des services, on remarque d'assez sensibles différences, le Royaume-Uni se distinguant par une croissance plus forte de l'emploi dans les services non publics, en particulier dans le secteur de la finance et des services aux entreprises, et plus faible dans les administrations publiques.

9 Ces tendances se sont-elles poursuivies au Royaume-Uni dans les années 1990 ?

Tableau 4. Croissance de l'emploi par secteur d'activité au Royaume-Uni, aux États-Unis et en France, 1979-1990 (en \% annuel moyen)

\begin{tabular}{|c|c|c|c|c|}
\hline \multicolumn{2}{|l|}{ Pays } & $\begin{array}{l}\text { Royaume- } \\
\text { Uni }\end{array}$ & $\begin{array}{l}\text { États- } \\
\text { Unis }\end{array}$ & France \\
\hline \multicolumn{2}{|l|}{ Agriculture } & $-1,6$ & $-0,5$ & $-3,7$ \\
\hline \multicolumn{2}{|c|}{ Industrie manufacturière } & $-2,9$ & $-0,5$ & $-1,6$ \\
\hline \multicolumn{2}{|l|}{ BTP } & 1,2 & 1,6 & $-1,2$ \\
\hline \multirow{5}{*}{$\begin{array}{l}\text { Services non } \\
\text { publics }\end{array}$} & Total & 2,8 & 2,7 & 1,7 \\
\hline & Commerce de gros et de détail & 1,3 & 1,9 & 0,8 \\
\hline & Transports et communications & $-0,1$ & 1,2 & 0,8 \\
\hline & Finance et service aux entreprises & 5,2 & 4,3 & 3,5 \\
\hline & $\begin{array}{l}\text { Services collectifs, sociaux et } \\
\text { personnels }\end{array}$ & 5,5 & 3,1 & 2,3 \\
\hline \multicolumn{2}{|c|}{ Administrations publiques } & 0,5 & 1,5 & 1,1 \\
\hline
\end{tabular}

Source: OCDE, Étude sur l'emploi, 1994, vol. 1, p. 3-4. 
Le tableau 5 illustre la répartition de l'emploi entre les trois grands secteurs d'activité et l'ampleur des créations ou suppressions d'emplois au sein de chacun d'entre eux de 1989 à 1999. On observe une régression de près de $0,2 \%$ en moyenne annuelle dans l'agriculture et de 1,7 \% dans l'industrie', et une progression de 1,3\% dans les services. La décennie se caractérise donc par un déclin moins rapide de l'emploi agricole et industriel que cela n'avait été le cas dans les années 1980, et par une progression elle aussi un peu moins rapide des emplois dans les services. Ainsi, si la tendance générale reste bien la même que pour la décennie antérieure, il apparaît néanmoins clairement que les écarts entre les évolutions sectorielles se sont quelque peu estompées dans les années 1990.

Tableau 5. Répartition de l'emploi au Royaume-Uni, par secteur d'activité, 1989-1999

\begin{tabular}{|c|c|c|c|c|c|c|}
\hline \multirow{2}{*}{ Secteurs } & \multicolumn{2}{|l|}{1989} & \multicolumn{2}{|l|}{1999} & \multicolumn{2}{|l|}{$\begin{array}{l}\text { Variation } \\
1989-99\end{array}$} \\
\hline & en milliers & en $\%$ & en milliers & en $\%$ & en milliers & en $\%$ \\
\hline Agriculture & 324 & 1,4 & 318 & 1,3 & -6 & $-1,8$ \\
\hline Industrie manufacturière & 4877 & 21,1 & 4041 & 16,8 & -836 & $-17,1$ \\
\hline Services & 16248 & 70,3 & 18380 & 76,4 & 2132 & 13,1 \\
\hline
\end{tabular}

Source : Employment Gazette, novembre 1989 ; Labour Market Trends, janvier 2001.

11 Les rôles respectifs du secteur privé et du secteur public dans la création d'emplois doivent également être soulignés (voir tableau 6). Ainsi, alors que près de 900000 emplois étaient supprimés dans le secteur public entre 1991 et 1999, le secteur privé en créait 1,6 millions.

Tableau 6. Répartition de l'emploi entre le secteur privé et le secteur public, 1991 et 1999

\begin{tabular}{|l|l|l|l|l|l|l|}
\hline \multirow{2}{*}{ Secteur } & \multicolumn{2}{|l}{1991} & \multicolumn{2}{l|}{1999} & \multicolumn{2}{l|}{$\begin{array}{l}\text { Variation } \\
1991-1999\end{array}$} \\
\cline { 2 - 7 } & en milliers & en \% & en milliers & en \% & en milliers & en \% \\
\hline Secteur privé & 21290 & 78,5 & 22889 & 82,2 & +1599 & $+7,5$ \\
\hline Secteur public & 5848 & 21,5 & 4967 & 17,8 & -881 & $-15,1$ \\
\hline
\end{tabular}

Source: Duncan Mc GREGOR, 'Jobs in the Public and Private Sectors', Economic Trends, N 559, juin 2000, p. 34.

12 On comprend dès lors pourquoi, au sein du secteur tertiaire, les sous-secteurs les plus fortement créateurs d'emplois ont été les services aux entreprises, le commerce, la santé et les services sociaux, ainsi que l'hôtellerie et la restauration (voir tableau 7). Il est cependant intéressant de relever que les services financiers, qui avaient été fortement créateurs d'emplois dans les années 1980, en ont perdu dans les années $1990^{10}$. Par ailleurs, le secteur qui a créé le plus grand nombre d'emplois dans les années 1990 a été la 
catégorie «activités de location, recherche, informatique et autres services aux entreprises ». C'est un point sur lequel nous reviendrons dans la troisième partie.

Tableau 7. Création d'emplois au Royaume-Uni dans les services, 1989-1999 (en milliers et en \%)

\begin{tabular}{|c|c|c|c|c|}
\hline \multirow{2}{*}{ Secteurs d'activité } & \multirow{2}{*}{1989} & \multirow{2}{*}{1999} & \multicolumn{2}{|c|}{ Variation } \\
\hline & & & $\begin{array}{l}\text { en } \\
\text { milliers }\end{array}$ & $\begin{array}{l}\text { en } \% \text { annuel } \\
\text { moyen }\end{array}$ \\
\hline $\begin{array}{l}\text { Activités de location, informatique recherche, et autres } \\
\text { services aux entreprises }\end{array}$ & 2278 & 3172 & 894 & 3,9 \\
\hline Commerce de gros et de détail & 3671 & 4070 & 399 & 1,1 \\
\hline Santé et travailleurs sociaux & 2291 & 2535 & 244 & 1,1 \\
\hline Hôtellerie et restauration & 1150 & 1369 & 219 & 1,9 \\
\hline $\begin{array}{l}\text { Autres services sociaux, collectifs } \\
\text { et aux personnes }\end{array}$ & 975 & 1156 & 181 & 1,8 \\
\hline Immobilier & 195 & 302 & 107 & 5,5 \\
\hline Administrations publiques & 1362 & 1456 & 94 & 0,7 \\
\hline Enseignement & 1835 & 1899 & 64 & 0,3 \\
\hline Transport et stockage & 912 & 948 & 36 & 0,4 \\
\hline Postes et télécommunications & 471 & 474 & 3 & 0,1 \\
\hline Intermédiation financière & 1046 & 1008 & -36 & $-0,3$ \\
\hline
\end{tabular}

Source : Labour Market Trends, janvier 2001.

\section{Quels emplois?}

13 A la lumière des débats qu'a suscités le développement, depuis la fin des années 1970, de formes atypiques d'emploi et le brouillage de la frontière autrefois bien définie entre activité et inactivité, il convient de s'interroger sur la typologie des emplois créés dans les années 1990. Nous évoquerons ainsi la répartition des créations d'emplois entre les hommes et les femmes, puis entre les salariés et les travailleurs indépendants. La part relative des emplois à temps plein et des emplois à temps partiel sera ensuite analysée, ainsi que la part des dispositifs de lutte contre le chômage (formation, stages) dans l'emploi total. Enfin, nous aborderons la question des niveaux de qualification et de rémunération correspondant aux emplois créés pendant la période étudiée et les importantes disparités régionales en matière de création d'emplois. 
Comme le montre le tableau 8, la création d'emplois a surtout bénéficié aux femmes, l'emploi féminin ayant progressé de près de $20 \%$ en 20 ans, alors que l'emploi masculin régressait, dans le même temps, de près de $2 \%$. Cette évolution est bien évidemment à mettre en relation avec la disparition massive d'emplois industriels qui a été relevée cidessus, ainsi qu'avec le développement de l'emploi à temps partiel que nous évoquerons ultérieurement. Elle n'est pas étrangère à l'élévation des qualifications de la maind'œuvre féminine, qui est elle-même liée à l'amélioration de sa formation, et aux meilleures performances des filles dans le système éducatif ${ }^{11}$.

Tableau 8. Emplois masculins et féminins, 1979 -1999

\begin{tabular}{|c|c|c|c|c|c|c|c|c|c|c|}
\hline & \multirow{2}{*}{\multicolumn{2}{|c|}{1979}} & \multirow{2}{*}{\multicolumn{2}{|c|}{1989}} & \multirow{2}{*}{\multicolumn{2}{|c|}{1999}} & \multicolumn{4}{|c|}{ Variation 1979-99 } \\
\hline & & & & & & & \multicolumn{2}{|c|}{ en milliers } & \multicolumn{2}{|c|}{ en $\%$} \\
\hline & $\mathrm{H}$ & F & $\mathrm{H}$ & $F$ & $\mathrm{H}$ & $F$ & $\mathrm{H}$ & $F$ & $\mathrm{H}$ & $\mathrm{F}$ \\
\hline En milliers & 15320 & 9975 & 15027 & 11122 & 15049 & 11916 & -271 & 1941 & $-1,8$ & 19,5 \\
\hline En $\%$ & 60,5 & 39,5 & 57,5 & 42,5 & 55,8 & 44,2 & - & - & - & - \\
\hline
\end{tabular}

Source : OCDE, Statistiques de la population active, 1999 ; Labour Market Trends, janvier 2001.

La question du développement du travail indépendant a aussi fait couler beaucoup d'encre, Margaret Thatcher et ses fidèles l'interprétant comme une manifestation du renouveau de l'esprit d'entreprise induit par les réformes mises en œuvre dans les années 1980, les adversaires du thatchérisme y voyant au contraire la manifestation d'une réalité plus inquiétante: beaucoup de chômeurs, las de ne pas trouver d'emploi salarié, n'auraient d'autre issue, pour trouver le moyen de gagner leur vie, que de s'établir à leur compte. Sans entrer dans le débat de fond - on peut cependant avancer que chacune de ces deux explications doit être prise en compte -, force est de constater la nette progression du travail indépendant (voir tableau 9), qui concerne surtout les hommes (à $72,4 \%$ en 1999 , contre $27,6 \%$ pour les femmes), et a donc plutôt favorisé l'emploi masculin.

Tableau 9. Progression du nombre des travailleurs indépendants, 1979-1999

\begin{tabular}{|l|l|l|l|l|l|}
\hline & \multirow{2}{*}{1979} & \multirow{2}{*}{1989} & \multirow{3}{*}{1999} & \multicolumn{2}{|l|}{$\begin{array}{l}\text { Variation } \\
1979-1999\end{array}$} \\
\cline { 4 - 6 } & & & & en milliers & en \% \\
\hline En milliers & 1906 & 3528 & 3203 & 1297 & 68,0 \\
\hline En \% du total des emplois & 7,6 & 13,2 & 11,7 & - & - \\
\hline
\end{tabular}

Source : OCDE, Statistiques de la population active, 1999, Labour Market Trends, janvier 2001. 

tassement dans les années 1990. Pour rendre compte de ce tassement, peut-être faut-il prendre en compte le faible taux de survie, au-delà de cinq années, des entreprises individuelles. On peut ainsi émettre l'hypothèse selon laquelle un nombre non négligeable de ceux qui avaient créé leur entreprise dans les années 1980 ont dû à nouveau chercher du travail dans les années 1990. La forte croissance enregistrée dans la seconde moitié de la décennie, accompagnée d'une accélération de la création d'emplois, a sans doute permis à bon nombre d'entre eux de retrouver un emploi salarié. Il est en effet significatif que ce soit à partir de 1997 que le tassement de l'emploi indépendant est observé ${ }^{12}$.

La question du travail à temps partiel est largement étudiée dans plusieurs des autres contributions au présent volume, à juste titre car son développement est l'une des principales caractéristiques de l'évolution contemporaine de l'emploi en GrandeBretagne.

Tableau 10. Emplois à temps partiel, 1983-1999

\begin{tabular}{|l|l|l|l|l|l|}
\hline & \multirow{2}{*}{1983} & \multirow{2}{*}{1992} & \multirow{2}{*}{1999} & \multicolumn{2}{|l|}{$\begin{array}{l}\text { Variation } \\
1983-1999\end{array}$} \\
\cline { 5 - 7 } & & & & en milliers & en \% \\
\hline En milliers & 4287 & 6018 & 6840 & 2553 & 59,5 \\
\hline En \% du total des emplois & 18,4 & 23,4 & 24,8 & - & - \\
\hline
\end{tabular}

Source : OCDE, Statistiques de la population active, 1999 ; Labour Market Trends, janvier 2001.

Tableau 11. Raisons données pour justifier d'une situation d'emploi à temps partiel au printemps 1999 (en \%)

\begin{tabular}{|l|l|l|}
\hline Réponse & Hommes & Femmes \\
\hline Ne voulaient pas un emploi à temps plein & 41,7 & 80,1 \\
\hline N'ont pu trouver un emploi à temps plein & 21,1 & 7,6 \\
\hline Poursuite d'études & 34,0 & 10,7 \\
\hline
\end{tabular}

Source : Regional Trends, 2000, p 83.

La lecture du tableau 10 nous permet de voir que c'est surtout dans les années 1980 que la progression de l'emploi à temps partiel a été forte (+ 5 points entre 1983 et 1992). La tendance s'est poursuivie dans les années 1990, mais à un rythme sensiblement moins élevé $(+1,4$ points de 1992 à 1999). Cette progression est un phénomène qui concerne principalement les femmes puisque celles-ci représentaient, en 1999, 80,3\% des employés à temps partiel. Notons toutefois que la proportion était de $85,1 \%$ en 1990 , ce qui montre que les hommes sont eux aussi de plus en plus concernés par cette tendance. Reflète-t- 
elle une dégradation du marché du travail, puisqu'un emploi à temps partiel est, par définition, moins rémunérateur qu'un emploi de même nature à plein temps, et qu'il comporte en outre généralement moins de garanties en termes de protection sociale? Pour tenter de répondre à cette question, sans doute convient-il de relever d'emblée que la très grande majorité des Britanniques qui travaillent à temps partiel le font volontairement: le temps partiel involontaire ne concerne qu'une minorité d'entre eux $(10,5 \% \text { en } 1999)^{13}$.

On relève aussi que la proportion des salariés en situation de temps partiel involontaire a baissé dans la seconde moitié de la décennie (elle était de 11,3\% en 1992 et 13,8\% en 1995), sans aucun doute parce que les créations d'emplois intervenues dans cette période leur ont plus facilement permis de trouver les emplois à plein temps auxquels ils aspiraient. En revanche, le développement de la pluriactivité reflète sans doute le fait que certains Britanniques qui n'ont pu trouver un travail à temps plein sont obligés d'occuper deux, voire trois emplois pour gagner leur vie. Notons toutefois que ce phénomène ne touchait que $4,7 \%$ des salariés en 1999. Le travail temporaire constitue une autre forme d'emploi atypique, censée traduire le développement de la précarité. Il touchait 7,4\% des salariés à l'été 2000 .

Un dernier point, souvent évoqué par ceux qu'inquiètent les évolutions contemporaines, est la part du "chômage déguisé » dans les chiffres de l'emploi. En effet, les individus suivant des stages ou des programmes de formation ('work-related training programmes') sont comptabilisés, depuis 1983, comme faisant partie de la population active occupée, alors que d'une part, ils ne sont pas des "salariés» au sens propre du terme et que d'autre part des études ont montré que ces dispositifs de formation étaient loin de toujours déboucher sur un emploi. L'inclusion de ces catégories dans la population active peut donc légitimement apparaître comme abusive. Elle a en tout cas pour effet mécanique de faire tout à la fois progresser les chiffres de l'emploi et diminuer ceux du chômage. Il convient toutefois de relativiser l'ampleur du phénomène, car si le nombre des bénéficiaires de ces programmes s'est fortement accru de 1983 à 1990 (passant alors de 9000 à 448 000, pour représenter en 1990 1,7\% de l'emploi total), il était retombé à 294000 au premier trimestre de 1995 et à 150000 au premier trimestre de 2000, soit 0,5\% de l'emploi total ${ }^{14}$. De surcroit, comme cela a été relevé plus haut et comme l'illustre le tableau 12, l'étude des niveaux de qualification auxquels correspondent les emplois créés révèle que les professions où l'emploi a le plus progressé sont les emplois qualifiés et bien rémunérés (cadres supérieurs, professions libérales). Certes, comme tous les pays développés, le Royaume-Uni compte ses 'Mc Jobs', mais leur proportion dans l'emploi total reste stable $(6 \%$ des hommes et $12 \%$ des femmes occupaient ainsi des emplois de vendeurs en 2000, chiffre inchangé par rapport à 1991).

Tableau 12. Répartition professionnelle de l'emploi salarié, 1991 et 2000 (en \%)

\begin{tabular}{|l|l|l|l|l|}
\hline \multirow{2}{*}{} & \multicolumn{2}{|l|}{ Hommes } & \multicolumn{2}{l|}{ Femmes } \\
\cline { 2 - 6 } & 1991 & 2000 & 1991 & 2000 \\
\hline Cadres supérieurs & 16 & 19 & 8 & 11 \\
\hline Professions libérales & 10 & 12 & 8 & 10 \\
\hline
\end{tabular}




\begin{tabular}{|l|l|l|l|l|}
\hline Cadres moyens et techniciens supérieurs & 8 & 9 & 10 & 11 \\
\hline Employés de bureau & 8 & 8 & 29 & 25 \\
\hline Travailleurs manuels qualifiés & 21 & 17 & 4 & 2 \\
\hline $\begin{array}{l}\text { Employés dans les services aux personnes, } \\
\text { la surveillance et la protection }\end{array}$ & 7 & 8 & 14 & 17 \\
\hline Vendeurs & 6 & 6 & 12 & 12 \\
\hline Travailleurs manuels sans qualification & 15 & 14 & 5 & 4 \\
\hline Autres & 8 & 8 & 10 & 8 \\
\hline
\end{tabular}

Source : Social Trends, 2001, p. 81

21 A toutes ces remarques il conviendrait d'ajouter que les créations d'emplois ont une forte dimension régionale, qui mériterait un examen approfondi auquel le format de cet article ne permet pas de se livrer. L'idée générale qui se dégage de l'examen des statistiques est qu'alors que les années 1980 avaient vu des pertes d'emplois massives dans le Nord industriel et une forte création d'emplois dans les services et les nouvelles technologies du Sud, il semble que l'écart entre le Nord et le Sud se soit légèrement atténué dans les années 1990. Le tableau 13 fait ainsi apparaître que le taux d'emploi a progressé partout, mais que l'écart vis-à-vis de la moyenne nationale a évolué différemment selon les régions depuis la fin de la récession du début des années 1990. Il a crû dans un sens négatif dans le Nord-Ouest, le Nord-Est, le Yorkshire et Humber, le pays de Galles et l'Écosse, ainsi qu'à Londres, ce qui traduit les difficultés particulières qu'ont rencontrées ces régions au regard de la création d'emplois. Il s'est en revanche creusé à l'avantage de l'Est, du Sud-Est et du Sud-Ouest, régions dont les taux d'emploi dépassaient largement la moyenne nationale en 2000 (de 4,7, 5,4 et 4,3 points respectivement), ce qui reflète bien leur dynamisme. L'atténuation de l'écart constaté vis-à-vis de la moyenne nationale par rapport à 1992 peut quant à lui refléter soit un dynamisme plus grand en termes de créations d'emplois, comme dans le cas des Midlands de l'Ouest, soit, au contraire, un certain émoussement du dynamisme observé antérieurement, comme dans le cas des Midlands de l'Est.

Tableau 13. La dimension régionale de la création d'emplois dans les années 1990

\begin{tabular}{|l|l|l|l|l|l|l|}
\hline \multirow{2}{*}{} & \multicolumn{2}{|l|}{$1992^{1}$} & \multicolumn{2}{l|}{$2000^{2}$} \\
\cline { 2 - 8 } & $\begin{array}{l}\text { Emploi en } \\
\text { milliers }\end{array}$ & $\begin{array}{l}\text { Taux } \\
\text { d'emploi } \\
\text { moyenne } \\
\text { nationale }\end{array}$ & $\begin{array}{l}\text { Écart avec la } \\
\text { Emploi en } \\
\text { milliers }\end{array}$ & $\begin{array}{l}\text { Taux } \\
\text { d'emploi }\end{array}$ & $\begin{array}{l}\text { Écart avec la } \\
\text { moyenne } \\
\text { nationale }\end{array}$ \\
\hline $\begin{array}{l}\text { Grande- } \\
\text { Bretagne }\end{array}$ & 24461 & 71,4 & 0 & 27292 & 74,8 & 0 \\
\hline Nord-Est & 1056 & 66,0 & $-5,4$ & 1090 & 67,9 & $-6,9$ \\
\hline
\end{tabular}




\begin{tabular}{|l|l|l|l|l|l|l|}
\hline Nord-Ouest & 2959 & 69,5 & $-1,9$ & 3101 & 72,5 & $-2,3$ \\
\hline $\begin{array}{l}\text { Yorkshire et } \\
\text { Humber }\end{array}$ & 2199 & 70,8 & $-0,6$ & 2342 & 74,0 & $-0,8$ \\
\hline $\begin{array}{l}\text { Midlands de } \\
\text { l'Est }\end{array}$ & 1870 & 73,5 & $+2,1$ & 2012 & 76,0 & $+1,2$ \\
\hline $\begin{array}{l}\text { Midlands de } \\
\text { l'Ouest }\end{array}$ & 2321 & 69,9 & $-1,5$ & 2456 & 73,5 & $-1,3$ \\
\hline Est & 2461 & 75,9 & $+4,5$ & 2737 & 79,5 & $+4,7$ \\
\hline Londres & 3062 & 68,0 & $-3,4$ & 3394 & 70,7 & $-4,1$ \\
\hline Sud-Est & 3692 & 76,0 & $+2,2$ & 4108 & 80,2 & $+5,4$ \\
\hline Sud-Ouest & 2128 & 73,6 & $+2,2$ & 2400 & 79,1 & $+4,3$ \\
\hline $\begin{array}{l}\text { Pays de } \\
\text { Galles }\end{array}$ & 1195 & 67,4 & $-4,0$ & 1261 & 69,5 & $-5,3$ \\
\hline \begin{tabular}{l} 
Écosse \\
\hline
\end{tabular} & 2304 & 71,1 & $-0,3$ & 2391 & 73,8 & $-1,0$ \\
\hline
\end{tabular}

1 mars-mai

${ }^{2}$ octobre-décembre

Source: Office for National Statistics, Labour Force Survey, Seasonally Adjusted Historical Supplement 1992-1999, 1999; Labour Market Trends, mars 2001.

Pour conclure cet état des lieux, on peut donc dire que les années 1990 ont été marquées par des créations nettes d'emplois moins fortes que pendant les années 1980, mais plus importantes qu'au cours des années 1960 et 1970. L'étude de la nature et de la typologie des emplois créés fait apparaître de fortes disparités, puisqu'à côté d'un grand nombre d'emplois très qualifiés et bien rémunérés, beaucoup des nouveaux emplois sont atypiques, qu'il s'agisse d'emplois à temps partiel ou d'emplois temporaires. Ces emplois atypiques représentaient un tiers environ de l'emploi total à la fin des années 1990 . Il n'est donc guère étonnant, dans ce contexte, que si la création d'emplois a incontestablement fait reculer le chômage, elle n'ait pas permis d'endiguer le creusement des inégalités sociales qui a caractérisé le Royaume-Uni depuis les années 1980. Mais la France et les États-Unis n'ont guère fait mieux dans ce domaine.

Il nous reste à essayer de comprendre pourquoi la création d'emplois a été beaucoup plus forte sur la période 1980-2000 qu'au cours des vingt années antérieures, et pourquoi la décennie 1990 marque néanmoins un reflux par rapport aux années 1980. 


\section{Les ressorts de la création d'emplois : quelques pistes de recherche}

\subsection{Croissance et emploi}

L'analyse keynésienne privilégie, on le sait, le rôle de la croissance dans la création d'emplois. Sur le court terme, ce facteur semble bien avoir joué dans le cas du RoyaumeUni où, comme partout, la création d'emplois a une forte dimension conjoncturelle. Il y a toujours contraction de la croissance de l'emploi, voire croissance négative de celle-ci, pendant les périodes de récession et pendant environ un an après le début de la phase de reprise. En revanche, les périodes de croissance vont de pair avec des créations nettes d'emplois. C'est bien ce qui s'est passé au Royaume-Uni dans les années 1990, puisque entre le printemps 1990 et le printemps 1993, 1,4 millions d'emplois nets ont été perdus, et que ce n'est qu'à partir du printemps 1993 que l'emploi a de nouveau augmenté, et ce jusqu'à la fin de la décennie ${ }^{15}$. Sur le long terme, en revanche, la relation entre le niveau de la croissance et le niveau des créations d'emplois semble, dans le cas du Royaume-Uni en tout cas, être moins fermement établie. En effet, sur la période 1980-2000, la croissance n'a guère été plus forte en moyenne (2,7 \%) que sur la période $1960-80$ (2,6 \%), et pourtant la création d'emplois s'est nettement accélérée à partir des années 1980 (voir tableau 2). La différence entre taux de croissance est donc un élément insuffisant pour rendre compte de l'accélération des créations d'emplois à laquelle on a assisté à partir des années 1980. On peut toutefois remarquer que, si on compare les années 1980 et les années 1990, le taux de croissance plus faible enregistré dans la seconde décennie (2,5\% contre 3,0\% pour la décennie précédente) peut expliquer le tassement relatif du rythme des créations d'emplois observé au cours de cette période ${ }^{16}$. Ce facteur n'est toutefois pas le seul à considérer.

\subsection{Les facteurs démographiques}

Le rythme d'accroissement de la population active, lui-même largement tributaire de l'accroissement de la population en âge de travailler (âgée de 16 à 64 ans), doit en effet être également pris en compte. Or cette dernière s'est accrue au taux annuel moyen de $0,5 \%$ de 1979 à 1989, puis de $0,3 \%$ de 1989 à 1999 . On constate toutefois que ces deux taux sont plus faibles que ceux concernant la croissance de l'emploi, qui se situent à $0,8 \%$ pour les années 1980 et $0,4 \%$ pour les années 1990 (voir tableau 2). Plus que l'accroissement de la population d'âge actif, c'est donc l'augmentation du taux d'activité, et en particulier du taux d'activité des femmes, qui a facilité la croissance de l'emploi ${ }^{17}$.

Il semble toutefois que les ressorts de la création d'emplois soient aussi à trouver ailleurs que dans le niveau de croissance de l'activité économique (la demande globale) ou dans l'accroissement de la population d'âge actif (l'offre de travail), et plus particulièrement dans les évolutions structurelles qui ont marqué l'économie britannique depuis les années 1980: réformes portant sur le fonctionnement du marché du travail (déréglementation, flexibilité), caractéristiques de l'économie de services, dynamisme de la création d'entreprises (en particulier, micro-entreprises ou PME-PMI), et évolution des pratiques managériales des grandes entreprises. 


\subsection{Déréglementation et flexibilité}

27 Les mesures mises en œuvre pendant les années 1980 pour augmenter la flexibilité sur le marché du travail britannique ont été abondamment étudiées et sont donc bien connues ${ }^{18}$ . L'argument économique majeur en faveur de la flexibilité est qu'elle renforce la capacité d'adaptation de l'offre de travail à la demande, et vice versa, et qu'elle abaisse le coût du travail. A contrario des réglementations excessives peuvent dissuader les employeurs d'embaucher. L'exemple le plus souvent cité est celui des réglementations limitant les licenciements : si elles sont trop restrictives, les entreprises hésiteront à embaucher en période de croissance, de crainte d'avoir des difficultés à licencier en cas de retournement de la conjoncture et de baisse de leur activité. Dans ce cas, les entreprises préfèreront délocaliser ou recourir à l'intérim ou à la sous-traitance. Si l'on suit ce raisonnement, on comprend pourquoi la déréglementation du marché du travail et la réduction systématique du pouvoir syndical dans l'entreprise opérées dans les années 1980 peuvent apparaître comme ayant "porté leurs fruits » en matière de créations d'emploi ${ }^{19}$. Dans cette perspective, il est toutefois intéressant de noter que la mise en place du salaire minimum, tout comme l'adoption de la directive européenne concernant le temps de travail, consécutives au retour au pouvoir des travaillistes à la fin des années 1990, n'ont pas entraîné les suppressions d'emplois que les conservateurs, hostiles à ces mesures, avaient annoncées ${ }^{20}$.

L'argument mis en avant par les adversaires de ces mesures était le renchérissement du coût du travail qu'elles allaient entraîner. Pour ce qui est du salaire minimum, on peut expliquer son absence d'effets négatifs en termes d'emplois en invoquant l'argument keynésien selon lequel il a relevé le pouvoir d'achat des salariés au bas de la pyramide des salaires et par là même soutenu la demande intérieure. Il convient également de noter que les travaillistes, soucieux de préserver les « acquis » des années 1979-1997 en termes de flexibilité du marché du travail, ont agi avec prudence et fixé le salaire minimum à un niveau suffisamment bas pour qu'il ne dissuade pas les employeurs d'embaucher. N'oublions pas, enfin, que l'introduction du salaire minimum eut lieu dans une période de croissance soutenue.

\subsection{La nature de l'économie de services}

Comme nous l'avons vu, plus de $76 \%$ des Britanniques travaillent aujourd'hui dans les services. Or, les gains de productivité dans ce secteur sont plus faibles que ceux des autres secteurs d'activité, ce qui signifie que son expansion a été plus créatrice d'emplois que ne l'aurait été l'expansion du secteur agricole ou industriel. Le Royaume-Uni ne fait pas ici exception à la règle qui veut que la demande de services croisse dans toutes les sociétés développées, du fait de facteurs sociaux comme le travail des femmes ou l'élévation du niveau de vie de la population. Les évolutions économiques et sociales qu'a connues ce pays depuis les années 1980 expliquent toutefois en partie pourquoi certaines catégories de services y ont progressé plus rapidement qu'ailleurs. Ainsi, une étude de John Philpott montre que l'enrichissement spectaculaire de certaines catégories de la population britannique (ceux que l'ont pourrait qualifier de "nouveaux riches du thatchérisme ») a entrainé une progression tout aussi spectaculaire de leur consommation en matière d'activités de loisirs (clubs de gymnastique, restaurants chics, etc.), et met en regard les 900000 emplois générés par le secteur de la restauration, ou les 350000 personnes 
employées le secteur des sports et loisirs à la fin des années 1990, et les 220000 salariés du secteur de la production d'énergie et du traitement et de la distribution de l'eau ${ }^{21}$.

Cet essor de l'économie de services a bien entendu profité tout particulièrement à la création d'emplois féminins, et ce à tous les niveaux de qualification requis dans ce secteur. Depuis les années 1970 les femmes bénéficient d'un accès accru à l'enseignement supérieur, et peuvent ainsi accéder aux métiers les plus qualifiés. Par ailleurs, à l'autre extrémité de la pyramide des salaires et des qualifications, elles sont souvent davantage prêtes que les hommes à accepter n'importe quel emploi, pourvu qu'il leur assure le revenu, ou le complément de revenu, dont elles ont besoin, car elles investissent généralement moins dans la réussite professionnelle que les hommes. Elles seraient enfin dotées de 'soft skills' (savoir-être) qui constituent des atouts pour certains emplois de service. N'oublions pas, enfin, que le secteur tertiaire permet aussi, beaucoup plus que les activités industrielles ou agricoles, le développement du travail à temps partiel dont nous avons vu qu'il était fortement créateur d'emplois depuis les années 1980, et qu'il bénéficiait plus particulièrement aux femmes.

\subsection{Le dynamisme de la création d'entreprises}

31 Encouragée pendant les années Thatcher et Major, la création d'entreprises, et notamment de petites entreprises, est elle aussi matière à controverse. Mais elle n'en demeure pas moins un fait certain ${ }^{22}$. Comme aux États-Unis et en France, ce sont ces PMEPMI qui sont le plus fortement créatrices d'emplois. L'emploi indépendant peut certes, comme nous l'avons déjà noté, constituer pour certains individus une façon de résoudre le problème du chômage. Mais les PME sont souvent plus efficaces en termes de coûts de production que des grandes entreprises (dont la taille conduit parfois à des déséconomies d'échelle), plus innovantes et plus flexibles. Elles peuvent donc embaucher davantage, surtout dans un pays comme le Royaume-Uni où elles savent que si la conjoncture se retourne, elles pourront aussi facilement licencier pour réduire leurs charges ${ }^{23}$. Au début de l'année 2000, 69,6\% de toutes les entreprises opérant au Royaume-Uni étaient des entreprises individuelles n'employant pas de salariés. Celles-ci sont particulièrement nombreuses dans le bâtiment, le commerce, l'immobilier, les services aux entreprises et aux particuliers, activités dont nous avons vu qu'elles étaient fortement créatrices d'emploi dans les années $1990^{24}$. Par ailleurs, à ces $69,6 \%$ s'ajoutaient $29,5 \%$ d'entreprises employant de 1 à 49 personnes. C'est donc au total 99,1\% des entreprises britanniques qui sont, au regard de la définition en vigueur au sein de l'Union européenne, des PME-PMI. Toutefois, la durée de vie des petites entreprises étant en moyenne plus courte que celles des grandes entreprises, le fait qu'elles interviennent pour une part importante dans la création d'emplois au Royaume-Uni pourrait avoir une incidence négative sur la stabilité de ces emplois. Il est donc quelque peu surprenant qu'en moyenne, la durée de l'emploi ( «job tenure ») n'ait guère varié au Royaume-Uni depuis 1975, même si cette moyenne cache de fortes disparités en fonction du sexe et de l'âge, disparités dont il serait intéressant de pouvoir cerner les origines ${ }^{25}$.

\subsection{L'évolution des pratiques managériales}

Après la vogue de la croissance conglomérale et de la recherche d'une taille maximale, censée permettre de réaliser des économies d'échelle, les grandes entreprises, depuis les 
années 1980, se sont de plus en plus recentrées sur leur métier de base ('core competencies' ), et ont de plus en plus externalisé toute une série d'activités moins essentielles, par le biais d'un recours accru à la sous-traitance. Cela n'est pas propre à la Grande-Bretagne, puisque ce bouleversement, venu des États-Unis, affecte aussi les autres pays européens, dont la France. C'est dans l'externalisation de multiples fonctions et le recours systématique à la sous-traitance que l'on retrouve l'origine des chiffres de la création d'emplois dans des activités comme la comptabilité, la communication, l'informatique, etc. dont on trouve la trace dans la rubrique "activités de location, informatique, recherche et autres services aux entreprises " figurant au tableau 7. C'est aussi là l'une des raisons de la prolifération des PME évoquée ci-dessus. La prise en compte de cette réalité doit d'ailleurs nous inviter à considérer les statistiques avec prudence, pour déjouer certains effets de trompe-l'œil. Si, en effet, une entreprise comme ICI décide d'externaliser son service juridique, elle supprimera des emplois, et l'opération sera comptabilisée comme une perte d'emplois industriels (ICI étant une entreprise industrielle, tous ses salariés sont recensés par les statistiques officielles comme des salariés de l'industrie). En revanche, le cabinet auquel elle sous-traitera dorénavant cette activité créera des emplois qui seront, eux, comptabilisés comme des emplois de service. En réalité, il y aura peut-être tout simplement eu transfert d'emplois de service d'une entreprise à une autre, mais ce n'est pas ainsi que se traduira l'opération statistiquement.

Sans doute serait-il présomptueux de vouloir conclure sur un sujet aussi vaste et complexe que la création d'emplois au Royaume-Uni dans les années 1990. Certains des phénomènes étudiés ici, notamment dans la deuxième partie, sont assez bien connus, et le lecteur averti aura sans doute eu l'impression de " déjà lu ». Rien, ou presque, n'a été dit des effets de la "nouvelle économie » sur la création d'emplois dans les années 1990, faute d'éléments aisément quantifiables sur le sujet, mais il s'agit là d'une piste de recherche qui mériterait d'être exploitée ${ }^{26}$. Il nous semble en revanche que dans la mesure où la réalité de l'accélération de la création d'emplois à partir des années 1980, et de son tassement relatif dans les années 1990, a été peu étudiée, il n'était pas inutile de se pencher tant sur le phénomène lui-même que sur les facteurs qui peuvent en rendre compte, au risque de heurter quelques idées reçues. Certes, ce bref article est loin d'épuiser la question et de résoudre l'ensemble des problèmes méthodologiques - en particulier statistiques - que requerrait une étude approfondie sur le sujet. Son ambition se limitait à montrer qu'il y a bien, en ce domaine, matière à recherche et à questionnement.

\section{BIBLIOGRAPHIE}

AZUELOS, Martine, L'économie du Royaume-Uni et des États-Unis depuis la fin des années 1970, Paris : PUF, 1994, chapitre VI, pp. 155-167.

BEDNARZIK, Robert W., 'The Role of Entrepreneurship in U.S. and European Job Growth', Monthly Labor Review, juillet 2000, pp. 3-16. 
BLANCHFLOWER, D. \& BURGESS, S. 'Job Creation and Job Destruction in the United Kingdom, 1980-1990', London School of Economics and Political Science, Centre for Economic Performance, Working Paper $N^{\circ}$ 515, septembre 1993.

BOWER, Caroline, 'Trends in Female Employment', Labour Market Trends, février 2001, pp. 107-11.

DAVIE, Anne, « Les effets du salaire minimum sur l'emploi et sur la flexibilité du travail au Royaume-Uni, 1997-2000», in Martine AZUELOS et Marie-Claude ESPOSITO (eds), Travail et emploi : l'expérience anglo-saxonne. Aspects contemporains, Paris : L'Harmattan, 2001, pp. 221-242.

Department of Employment, Employment Gazette, mensuel. A été remplacé en 1995 par Labour Market Trends.

DICKENS, Richard, WADSWORTH, Jonathan \& GREGG, Paul (eds.), The State of Working Britain. Update 2001, London School of Economics and Political Science, Centre for Economic Performance, 2001, $64 \mathrm{p}$.

DICKENS, Richard, WADSWORTH, Jonathan \& GREGG, Paul, 'Overview of the British Labour Market in Recovery', in DICKENS, Richard, WADSWORTH, Jonathan \& GREGG, Paul (eds.), The State of Working Britain. Update 2001, London School of Economics and Political Science, Centre for Economic Performance, 2001, pp. 1-7.

ESPOSITO, Marie-Claude. «Les PME-PMI et la restructuration industrielle au Royaume-Uni » in Martine AZUELOS (ed), Le modèle économique anglo-saxon à l'épreuve de la globalisation, Paris : Presses de la Sorbonne Nouvelle, 1996, pp. 73-90.

GAUSON, Helen, 'People and Jobs: Comparing Sources of Employment Data', Labour Market Trends, janvier 2002, pp. 29-32.

JOHNSON, Christopher, The Economy under Mrs Thatcher, Harmondsworth: Penguin, 1992, chapitre 7, pp. 217-249.

KARNEL, Solomon M. \& BRYON, Justin, A Comparison of Small and Medium Sized Enterprises in Europe and the USA, London: Routledge, 2002.

Mc GREGOR, Duncan, 'Jobs in the Public and Private Sectors', Economic Trends, N 559, juin 2000, pp. 33-38.

MISHEL, Lawrence, BERNSTEIN, Jared \& SCHMITT, John. The State of Working America. Washington, DC: Economic Policy Institute. Annuel.

OCDE, Economic Survey, United Kingdom, Paris: OCDE, 2000.

OCDE, Étude sur l'emploi, 3 vol. , Paris : OCDE, 1994.

Office for National Statistics, Economic Trends Annual Supplements, London: The Stationery Office, annuel.

Office for National Statistics, Labour Force Survey Seasonally Adjusted Historical Supplement, 1984-1998, London: The Stationery Office, 1999.

Office for National Statistics, Labour Force Survey Seasonally Adjusted Historical Supplement, 1992-1999, London: The Stationery Office, 1999.

Office for National Statistics, Labour Force Survey Quarterly Supplement, London: The Stationery office, trimestriel.

Office for National Statistics, Labour Market Trends, London: The Stationery Office, mensuel. A succédé en 1995 à l'Employment Gazette.

Office for National Statistics, Regional Trends, London: The Stationery Office, annuel. 
Office for National Statistics, Social Trends, London: The Stationery Office, annuel.

PHILPOTT, John, ‘Behind the Buzzword. The New Economy', Economic Report, The Employment Policy Institute, vol. 14, N 8, février 2000, 17 p.

TWOMEY, Breda, 'Women in the Labour Market: Results from the Spring 2001 LFS', Labour Market Trends, mars 2002, pp. 109-127.

United States Department of Labor, Monthly Labor Review, mensuel.

\section{NOTES}

1. La terminologie « Royaume-Uni » sera ici préférée à celle de « Grande-Bretagne » à chaque fois que les études ou les statistiques auxquelles on se référera porteront effectivement sur cet ensemble géographique.

2. OCDE, Economic Survey, United Kingdom, Paris : OCDE, 2000, p. 93. La stratégie pour l'emploi définie par ce même organisme en 1997 est fondée sur le développement de la flexibilité, des incitations au travail et sur l'élévation des qualifications de la main-d'œuvre.

3. Pour s'élever à nouveau à partir de cette date.

4. Sur ces évolutions, voir par exemple les études publiées régulièrement sur ce thème dans la Monthly Labor Review ou les dernières éditions de l'étude annuelle de Lawrence MISHEL et al. The State of Working America. Washington, DC: Economic Policy Institute.

5. Voir sur ce point la contribution de Marie-Annick MATTIOLI au présent volume.

6. Sur la comptabilisation des chiffres de l'emploi, on pourra se reporter à l'article de Helen GAUSON, "People and Jobs: Comparing Sources of Employment Data », Labour Market Trends, janvier 2002, pp. 29-32. Les chiffres utilisés pour le présent article sont extraits de Labour Market Trends ou de Social Trends pour les données relatives aux années 1990. Pour les séries plus longues, on s'est référé aux données fournies dans l'article publié en juin 2000 dans Economic Trends par Duncan Mc GREGOR (de la Division Emploi, Revenus et Productivité de l'Office for National Statistics). En effet, cet article fait état des révisions auxquelles a procédé l'ONS en 2000 pour les séries couvrant la période 1959-1995, à partir des données initialement fournies par l'enquête sur la population active (Labour Force Survey). Il faut toutefois relever que les données figurant dans une publication de référence comme The State of Working Britain Update 2001, fondées sur les « calculs des auteurs » à partir de la LFS, diffèrent notoirement de celles figurant dans les statistiques officielles (voir Richard DICKENS et al., 'An Overview of the British Labour Market in Recovery', in Richard DICKENS et al. (eds), The State of Working Britain. Update 2001, Centre for Economic Performance, London School of Economics and Political Science, Londres : 2001). En effet, d'une part, cette publication ne prend en compte que les données relatives à la GrandeBretagne, alors que les statistiques de l'ONS se réfèrent à la totalité du Royaume-Uni. Mais par ailleurs, elle semble minimiser singulièrement l'ampleur de la création d'emplois depuis 1979. En l'absence d'explications sur la façon dont les statistiques officielles ont été retravaillées par les économistes du Centre for Economic Performance, et eu égard au caractère très peu détaillé de leurs données concernant les créations d'emplois (un seul tableau, p. 2), il nous a donc paru plus prudent de nous référer aux seules statistiques officielles.

7. Les chiffres concernant l'évolution de l'emploi se réfèrent à la catégorie que les statistiques officielles, publiées par l'office for National Statistics, qualifient de 'workforce in employment' (la population active occupée) et qui comprend les salariés ('employee jobs'), les travailleurs indépendants ('self employed'), les militaires ('HM Forces'), et les individus suivant un programme de formation financé par les pouvoirs publics ('government supported trainees'). 
8. D. BLANCHFLOWER \& S. BURGESS, 'Job Creation and Job Destruction in the UK, 1980-90', London School of Economics and Political Science, Centre for Economic Performance, Working Paper $\mathrm{N}^{\circ}$ 515, London, September 1973.

9. En 1981, l'industrie manufacturière employait un salarié britannique sur trois. En 2001, la proportion était tombée à $22 \%$ (Source : Office for National Statistics, Social Trends 32, London: The Stationery Office, 2002, tableau 4.13, p. 76).

10. Voir sur ce point la contribution de Marie-Claude ESPOSITO au présent volume.

11. Tous ces phénomènes ont fait l'objet de nombreuses études. Voir en particulier, Caroline BOWER, 'Trends in Female Employment', Labour Market Trends, février 2001, pp. 107-118; ou Breda TWOMEY, 'Women in the Labour Market: Results from the Spring 2001 LFS', Labour Market Trends, mars 2002, pp. 109-127.

12. Voir Duncan Mc GREGOR, 'Jobs in the Public and Private Sectors', Economic Trends, $\mathrm{N}^{\circ} 559$, juin 2000 , tableau C, p. 37.

13. Certes, on pourrait souhaiter que des enquêtes de nature sociologique permettent de mieux cerner le sens des termes « volontaire » et « involontaire » dans ce contexte. Une femme avec des enfants, confrontée à des problèmes de garde dont on sait combien ils sont particulièrement difficiles en Grande-Bretagne, répondra qu'elle a " volontairement » choisi de travailler à temps partiel plutôt qu'à temps plein. Mais ce choix ne reflète-t-il pas le poids des contraintes socioéconomiques qui pèsent sur elle?

14. Chiffres donnés dans Economic Trends Annual Supplement, 2000. Le chiffre de l'année 2000 comprend les bénéficiaires du New Deal.

15. Voir Office for National Statistics, Labour Force Survey Seasonally Adjusted Historical Supplement, 1984-1998, London: The Stationery Office, 1999. On rappelle que le Royaume-Uni a connu une récession du troisième trimestre 1990 au deuxième trimestre 1992.

16. Taux de croissance moyens calculés par l'auteur à partir de Office for National Statistics, Economic Trends Annual Supplement 2001, London: The Stationery Office, tableau 1.1.

17. Le taux d'activité des femmes âgées de 16 à 59 ans est passé de 66,1\% en 1984 à $71,3 \%$ en 1990 et $73,0 \%$ en 2000 . Ce taux se définit comme la proportion des femmes de cette catégorie d'âge appartenant à la population active.

18. Voir, par exemple, Christopher JOHNSON, The Economy under Mrs Thatcher, Harmondsworth : Penguin, 1991, chapitre 7; ou, pour une présentation plus succincte, Martine AZUELOS, L'économie du Royaume-Uni et des États-Unis depuis la fin des années 1970, Paris : PUF, 1994, chapitre VI, pp. 155-167.

19. Voir supra, OCDE, note 2 .

20. Voir Anne DAVIE, «Les effets du salaire minimum sur l'emploi et sur la flexibilité du travail au Royaume-Uni, 1997-2000», in Martine AZUELOS et Marie-Claude ESPOSITO (eds), Travail et emploi : l'expérience anglo-saxonne. Aspects contemporains, Paris : L'Harmattan, 2001, pp. 221-242.

21. John PHILPOTT, 'Behind the Buzzword. The New Economy', Economic Report, The Employment Policy Institute, vol. 14, $\mathrm{N}^{\circ} 8$, février 2000, p.9.

22. Voir Marie-Claude ESPOSITO, «Les PME-PMI et la restructuration industrielle au RoyaumeUni » in Martine AZUELOS (ed), Le modèle économique anglo-saxon à l'épreuve de la globalisation, Paris : Presses de la Sorbonne Nouvelle, 1996, pp. 73-90.

23. Voir Robert W. BEDNARZIK, 'The Role of Entrepreneurship in U.S. and European Job Growth', Monthly Labor Review, juillet 2000, pp. 3-16, et Solomon M. KARNEL \& Justin BRYON, A Comparison of Small and Medium Sized Enterprises in Europe and the USA, London: Routledge, 2002.

24. Voir 'Labour Market Spotlight. Small and Medium Entreprises', Labour Market Trends, avril 2002, p. 173.

25. Voir Social Trends, 2002, p. 82.

26. Voir sur ce point l'étude déjà citée de John PHILPOTT, 'Behind the Buzzword. The New Economy', Economic Report, The Employment Policy Institute, vol. 14, $\mathrm{N}^{\circ} 8$, février 2000, $17 \mathrm{p}$. 


\section{RÉSUMÉS}

La création d'emplois en Grande-Bretagne dans les années 1990 a été tantôt ignorée, tantôt brandie comme la preuve éclatante du succès des réformes mises en œuvre depuis les années 1980, mais finalement peu étudiée. L'article commence par mesurer l'ampleur du phénomène et le replace dans le contexte des évolutions observables depuis les années 1960. Il met ainsi en évidence le volume de la création d'emplois, son rythme de progression, mais aussi l'évolution de la typologie des emplois créés, avant d'identifier les principaux facteurs qui permettent d'en rendre compte.

Job creation in Britain in the 1990s has been either altogether ignored or else celebrated as evidence of the success of the reforms implemented since the early 1980s. But, so far, little work has been devoted to the study and interpretation of available data on the subject. This article first examines the size and pace of employment growth in the 1990s, setting these against the backdrop of longer term trends. It then focuses on the major sectoral shifts and changing profiles of jobs in contemporary Britain, and identifies various factors accounting for faster job growth.

\section{AUTEUR}

\section{MARTINE AZUELOS}

Université de la Sorbonne Nouvelle-Paris III 